\title{
TREES WITH UNIQUE MINIMUM DOMINATING SETS
}

\author{
Sharada B \\ Department of Studies in Computer Science, University of Mysore, Manasagangothri, \\ Mysore
}

\begin{abstract}
A set $D$ of vertices of a graph $G$ is a dominating set if every vertex in $V \backslash D$ is adjacent to some vertex in $D$. In this paper, we provide a constructive characterization of trees with unique minimum dominating set.
\end{abstract}

\section{KEYWORDS}

Dominating set, graph, tree, vertex

\section{INTRODUCTION}

Let $G=(V, E)$ be a graph. The subset $D$ of $V$ is a dominating set if every vertex in $V \backslash D$ is adjacent to some vertex in $D$. The domination number of $G$, denoted by $\gamma(G)$, is the minimum cardinality of a dominating set of $G$. A dominating set of cardinality $\gamma(G)$ is called a $\gamma(G)$-set.

The literature on domination and its variations in graphs has been surveyed and detailed in the two books by Haynes, Hedetniemi, and Slater [1, 2]. Gunther, Hartnell, Markus, and Rall [3] have studied graphs with unique minimum dominating sets. Later, Fishermann and L. Volkzmann [4], made a thorough study of unique minimum domination in trees. Haynes and Henning [5] gave a characterization for trees with unique minimum total dominating sets. Chellali et. al[6], made a study on trees with unique minimum paired-dominating sets. Recently, Blidia et. al.[7] characterized the trees with unique minimum locating dominating sets. We provide a constructive characterization of trees with unique minimum dominating sets.

A graph $G$ is called a unique minimum domination graph, or just a UMD-graph, if $G$ has a unique $\gamma(G)$-set. Observe that the path $P_{3}$ has the support vertex set as its unique minimum dominating set. A vertex of degree one is called an end vertex or a leaf and its neighbour is called a support vertex. The set of leaves in $T$ is denoted by $L(T)$ and the set of support vertices by $S(T)$. For a vertex $v$ the open neighborhood of $v$ is the set $\mathrm{N}(v)$ and its closed neighborhood is the set $\mathrm{N}[v]$. We denote $G^{-v}$ as $G \backslash\{v\}$. For notation and graph theory terminology, we in general follow [8].

\section{KNOWN RESULTS}

The following is an important result which guides the main results of this paper. 
International Journal of Soft Computing, Mathematics and Control (IJSCMC), Vol. 4, No. 1, February 2015

\section{Theorem 2.1:}

Let $T$ be a tree of order $n \geq 3$. Then the following conditions are equivalent:

i. $\quad T$ is a UMD-tree with $\gamma(T)$-set $S$.

ii. $\quad T$ has a $\gamma(T)$-set $S$ for which every vertex $v \in S$ is a support vertex or satisfies $|\operatorname{pn}(v, S)| \geq$ 2.

iii. $\quad$ Thas a $\gamma(T)$-set Sfor which $\gamma(T-v)>\gamma(T)$ for every $v \in S$.

\section{MAIN RESULTS}

In this section we provide a constructive characterization of all UMD-trees. For this purpose we describe a procedure for building a family $\mathfrak{I}$ of labelled trees that have unique minimum dominating sets, as follows.

Let $\mathfrak{I}=\left\{T_{k}\right\}, k \geq 1$ be the family of trees constructed inductively such that $T_{1}$ is a star $K_{l, n}, n>1$ and $T_{k}=T$, a tree. If $k \geq 2, T_{i+l}$ can be obtained recursively from $T_{i}$ by the operation $\mathfrak{I}_{1}$ or $\mathfrak{I}_{2}$ for $i$ $=1,2, \ldots, k-1$.

We define the status of a vertex $v$, denoted $\operatorname{sta}(v)$ to be A or B. Initially if $T_{1}=K_{1, n}, n>1$, then $\operatorname{sta}(v)=\mathrm{A}$ if $v$ is a support vertex and $\operatorname{sta}(v)=\mathrm{B}$, if $\mathrm{v}$ is a leaf. Once a vertex is assigned a status, the status remains unchanged as the tree is constructed.

Operation $\mathfrak{\Im}_{1}$ : Assume $v \in T_{i}$ and $\operatorname{sta}(v)=\mathrm{A}$. The tree $T_{i+1}$ is obtained from $T_{i}$ by adding a star $K_{l, m}, m>1$ with support vertex $u$ and the edge $u v$. Let $\operatorname{sta}(u)=\mathrm{A}$ and $\operatorname{sta}(w)=\mathrm{B}$, where $w$ is a leaf of $K_{l, m}$.

Operation $\mathfrak{\Im}_{2}$ : Assume $v \in T_{i}$ and $\operatorname{sta}(v)=\mathrm{B}$. The tree $T_{i+1}$ is obtained from $T_{i}$ by adding a star $K_{l, m}, m>1$ with support vertex $u$ and the edge $u v$. Let $v$ be adjacent to $x$ in $T_{i}$. If $\operatorname{deg}(x)>2$ then $u$ is a support vertex of $K_{l, m}$. If $\operatorname{deg}(x)=2$ then $u$ is a leaf of $K_{l, m}$. Let $\operatorname{sta}(w)=\mathrm{A}$ if $w$ is a support vertex of $K_{l, m}$ and $\operatorname{sta}(w)=\mathrm{B}$, if $w$ is a leaf of $K_{l, m}$.

$\mathfrak{I}$ is closed under the two operations $\mathfrak{I}_{1}$ and $\mathfrak{I}_{2}$. For $T \in \mathfrak{I}$, let $\mathrm{A}(T)$ and $\mathrm{B}(T)$ be the sets of vertices of status A and B respectively. We have the following observations, which follow from the construction of $\Im$.

\section{Observations 3.1:}

Let $T \in \mathfrak{I}$, and $v \in V(T)$

i. If $\operatorname{sta}(v)=A$, then $v$ is adjacent to at least two vertices of $B(T)$.

ii. If $\operatorname{sta}(v)=B$, then $v$ is adjacent to exactly one vertex of $A(T)$.

iii. If $v$ is a support vertex, then $\operatorname{sta}(v)=A$.

iv. If $v$ is a leaf, then $\operatorname{sta}(v)=B$.

v. $|A(T)|<|B(T)|$. 
International Journal of Soft Computing, Mathematics and Control (IJSCMC), Vol. 4, No. 1, February 2015

\section{Lemma 3.2:}

If $T \in \mathfrak{I}$, then $A(T)$ is a $\gamma(T)$-set. Moreover if $T$ is obtained from $T^{\prime} \in \mathfrak{I}$, by the operation $\mathfrak{I}_{1}$ or $\mathfrak{I}_{2}$ then $\gamma(T)=\gamma\left(T^{\prime}\right)+1$.

\section{Proof:}

By the Observations, it is clear that $\mathrm{A}(T)$ is a dominating set. Now we prove that $\mathrm{A}(T)$ is a $\gamma(T)$ set. We proceed by induction on the length $k$ of the sequence of trees needed to construct the tree $T$. Suppose $k=1$, then $T=K_{l, n}, n>1$, belongs to $\mathfrak{I}$. Then by the construction of the family $\mathfrak{I}$, the only vertex of $\operatorname{sta}(\mathrm{A})$ is the support vertex of the star, which dominates all the other vertices. Hence, $\mathrm{A}(T)$ is a $\gamma(T)$-set. This establishes the base case.

Assume then that the result holds for all trees in $\mathfrak{I}$, that can be constructed from a sequence of fewer than $k$ trees where $k \geq 2$. Let $T \in \mathfrak{I}$ be obtained from a sequence $T_{1}, T_{2}, \ldots, T_{k}$ of trees, where $T^{\prime}=T_{k-1}$ and $T=T_{k}$. By our inductive hypothesis $\mathrm{A}(T)$ is a $\gamma(T)$-set.

We now consider two possibilities depending on whether $T$ is obtained from $T$ ' by the operation $\mathfrak{I}_{1}$ or $\mathfrak{I}_{2}$

Case 1: $T$ is obtained from $T^{\prime}$ by operation $\mathfrak{I}_{1}$.

Suppose $T$ is obtained from $T^{\prime}$ by adding a star $K_{l, m}, m>1$ with support vertex $u$ and the edge $u v$, where $v \in T$. Then $\operatorname{sta}(u)=\mathrm{A}$ and $\operatorname{sta}(w)=\mathrm{B}$, where $w$ is a leaf of $K_{l, m}$. Any $\gamma\left(T^{\prime}\right)$-set can be extended to a $\gamma(T)$-set by adding to it the support vertex $w$, which is of status A. Hence, $A(T)=A\left(T^{\prime}\right) \cup\{w\}$ is a $\gamma(T)$-set.

Since $T$ is obtained from $T$ ' by operation $\Im_{1}, T$ can have exactly one more vertex of status A than $T^{\prime}$. Since $\gamma(T)=|\mathrm{A}(T)|$ and $\gamma\left(T^{\prime}\right)=\left|\mathrm{A}\left(T^{\prime}\right)\right|$, it follows that $\gamma(T)=\gamma\left(T^{\prime}\right)+1$.

Case 2: $T$ is obtained from $T^{\prime}$ by operation $\mathfrak{I}_{2}$.

The proof is very similar to Case 1 .

\section{Lemma 3.3:}

If $T \in \mathfrak{I}$, then $\gamma(T-x)>\gamma(T)$, for every $x \in A(T)$.

\section{Proof:}

We proceed by induction on the order $p$ of $T$. If $p=3$ then $T=P_{3} \in \mathfrak{I}$. Let $P_{3}$ be labelled as $u, v$, $w$ i.e. $v$ is a support vertex, $u$ and $w$ are leaves. By previous lemma $\mathrm{A}\left(P_{3}\right)=\{v\}$ is a $\gamma\left(P_{3}\right)$-set. Clearly, $\gamma\left(P_{3}-v\right)=2>1=\gamma\left(P_{3}\right)$. This establishes the base case.

Assume that the lemma is true for $T^{\prime} \in \mathfrak{I}$ with $\left|\mathrm{V}\left(T^{\prime}\right)\right|<p$. Let $T=T_{r}$ be obtained from a sequence of trees $T_{1}, T_{2}, \ldots, T_{r-1}=T^{\prime}$. Since $\left|\mathrm{V}\left(T^{\prime}\right)\right|<|\mathrm{V}(T)|$, by induction hypothesis $\gamma\left(T^{\prime}-x\right)>\gamma\left(T^{\prime}\right)$, for every $x \in A\left(T^{\prime}\right)$. Two cases arise. 
Case 1: $x \in A\left(T^{\prime}\right)$.

Any $\gamma\left(T^{\prime}-x\right)$-set can be extended to a $\gamma(T-x)$-set by adding a vertex $y \in A(T) \backslash A\left(T^{\prime}\right)$ i.e. $\gamma(T-x)=\gamma\left(T^{\prime}-x\right)+1$. By previous lemma, $\gamma(T)=\gamma\left(T^{\prime}\right)+1<\gamma\left(T^{\prime}-x\right)+1=\gamma(T-x)$, for every $x \in A(T)$.

Case 2: $x \in A(T) \backslash A\left(T^{\prime}\right)$

Let $T$ be obtained from $T^{\prime}$ by adding a star $K_{l, m}, m>1$ to a vertex of $T^{\prime}$. Let $x$ be the support vertex of $K_{1, m} . T-x$ contains $m+1$ components, hence $\gamma(T-x)=\gamma\left(T^{\prime}\right)+m=\gamma(T)-1+m>\gamma(T)$, since $m>1$, as desired.

\section{Theorem 3.4:}

A tree $T$ has the unique minimum dominating set if and only if $T \in \mathfrak{J}$.

\section{Proof:}

By lemma 3.3, it is sufficient to prove that the condition is necessary. We proceed by induction on the order $p$ of the tree. For $p=3, T=P_{3}$ has the unique minimum dominating set and also $T \in \mathfrak{I}$. Assume that $p \geq 4$ and all trees $T$ of order less than $p$, with unique minimum dominating set belong to the family $\mathfrak{\Im}$.

Let $P: v_{1}, v_{2}, \ldots, v_{r}$ be a longest path in $T$. Obviously $\operatorname{deg}\left(v_{1}\right)=\operatorname{deg}\left(v_{r}\right)=1$ and $v_{2}$ is a support vertex. If it is a strong support vertex, let $v_{2}$ be adjacent to the leaves $l_{1}, l_{2}, \ldots, l_{\mathrm{s}}$. Then $K_{1, s}$ is a star with support vertex $v_{2}$. Define $T=T-K_{1, s}$. If $v_{2}$ is not a strong support vertex and $\operatorname{deg}\left(v_{2}\right)=2$ define $T^{\prime}=T \backslash\left\{v_{1}, v_{2}, v_{3}\right\}$. In both the cases, any $\gamma\left(T^{\prime}\right)$-set can be extended to a $\gamma(T)$-set by adding the vertex $v_{2}$. Hence, $\gamma(T)=\gamma\left(T^{\prime}\right)+1$.

Since $T$ is a UMD-tree, there exists a $\gamma(T)$-set $S$ such that $\gamma(T-x)>\gamma(T)$, for every $x \in S$. Let $S$ ' $=S \cap T^{\prime}$. Then $S^{\prime}$ is a $\gamma\left(T^{\prime}\right)$-set. Let $w \in S^{\prime}$ then $w \in S$ and hence $\gamma(T-w)>\gamma(T)$. Therefore $\gamma\left(T^{\prime}\right)$ $=\gamma(T)-1<\gamma(T-w)-1$.

Any $\gamma\left(T^{\prime}-w\right)$-set can be extended to a dominating set of $T-w$ by adding $v_{1}$. Thus $\gamma(T-w) \leq$ $\gamma\left(T^{\prime}-w\right)+1$. Hence $\gamma\left(T^{\prime}\right)<\gamma(T-w)-1 \leq \gamma\left(T^{\prime}-w\right)$, for every $w \in S$. Hence $T^{\prime}$ is a UMD-tree. Since $\left|\mathrm{V}\left(T^{\prime}\right)\right|<|\mathrm{V}(T)|$, by the induction hypothesis $T^{\prime} \in \mathfrak{I}$. Thus $T$ is obtained from $T^{\prime}$ by the operation $\mathfrak{I}_{1}$ or $\mathfrak{I}_{2}$. Hence $T \in \mathfrak{I}$.

\section{REFERENCES}

[1] Haynes T W, Hedetniemi S T \& Slater P J, (1998) Fundamentals of Domination in Graphs, Marcel Dekker, New York.

[2] Haynes T W, Hedetniemi S T \& Slater P J, (1998) (Eds.), Domination in Graphs: Advanced Topics, Marcel Dekker, New York.

[3] Gunther G, Hartnell B, Markus L R \& Rall D, (1994) "Graphs with unique minimum dominating Sets", Congr. Numer. Vol. 101, pp55-63.

[4] Fishermann M \& Volkmann L, (2002) "Unique minimum domination in trees", Aus. J. Comb. Vol. 25, pp117-124.

[5] Haynes T W, Henning M A, (2002) “Trees with unique total dominating sets”, Discuss. Math. Graph Theory, Vol. 22, pp233-246. 
International Journal of Soft Computing, Mathematics and Control (IJSCMC), Vol. 4, No. 1, February 2015

[6] Chellali M \& Haynes T W, (2004) "Trees with unique minimum paired-dominating sets", Ars Combin., Vol.25, pp3-12.

[7] Blidia M, Chellali M, Lounes R \& Maffray F, (2011) "Characterizations of trees with unique minimum locating-dominating sets", J. Combin. Math. and Combin. Comp., Vol.1, pp.23-33.

[8] West D B (2001) Introduction to graph theory, Prentice Hall.

\section{Authors}

Sharada B received the MCA (Computer Science), M.Sc. (Mathematics), Ph.D. degree from the University of Mysore, Mysore in 2002, 2004 and 2009 respectively. She is serving as Assistant Professor of Computer Science at niversity of Mysore, Manaagangothri, Mysore. Her current research interest includes Combinatorics, Graph Theory and Image Processing. 